\title{
Laser Treatment of Oral Benign Lesions: A Review about 4 Case Reports
}

\author{
Akram Belmehdi ${ }^{1}$, Karima El Harti ${ }^{2}$, Wafaa El Wady ${ }^{3}$ \\ ${ }^{I}$ Resident In The Department Of Oral Surgery, Dental Center Of Treatment And Diagnosis (Ibn Sina Hospital), \\ Rabat, Morocco \\ ${ }^{2}$ Professor Of Oral Surgery, Faculty Of Dentistry Of Rabat, Mohammed V University, Morocco. \\ ${ }^{3}$ Professor And Head Of The Oral Surgery Service, Faculty Of Dentistry Of Rabat, Mohammed V University,
} Morocco

\begin{abstract}
Laser technology has made a tremendous progress over few past decades, and lasers have found a niche in many surgical specialties. Lasers offer many useful clinical applications for oral and maxillofacial surgeons in the diagnosis and treatment of patients with different type of oral mucosal lesions and maxillofacial disorders, as long as the clinician receives the proper training to use this technology safely and effectively. The present paper reviews the different types of lasers and discusses their use a vital tool in excision of benign oral lesions with histological findings.
\end{abstract}

Keywords: Biopsy, benign oral lesions, diode laser, histological examination.

\section{Introduction}

Laser is an advanced technology widely applied in medicine and dentistry, minimally invasive to tissue, and allowing excellent wound healing. The recently rapid developments in laser technology and better understanding of bio-interactions of different laser systems have broaden the clinical use of laser in dentistry [12]. More than in any other dental specialty, lasers have played an integral role in the practice of oral surgery. They are rapidly becoming the standard of care for many procedures performed by oral and maxillofacial surgeons. The reason for this transition is due to the fact that many procedures can be executed more efficiently and with less morbidity using lasers as compared to a scalpel, electrocautery or high frequency devices [3-4-5].

This tool can treat almost all soft tissue injuries of medium-small size and realize tissue corrections with excellent postoperative results. This minimally invasive method does not require sutures and allows shortening duration of treatment, which represents a decisive benefit for the patient [6-7-8].

Through clinical case reports of oral mucosa benign tumors treated by the laser, we will highlight the features of this technique, its effects on living tissue, and discuss its current clinical applications.

\section{Surgical procedures:}

\section{Case reports}

We used Wiser dental diode laser from "Doctor Smile lasers" with a $980 \mathrm{~nm}$ diameter glass fiber as a delivery system.

Our surgical procedures were started with injection of the surgical site by local anesthesia. After anesthetized the surgical site, the lesion or the mass grasped with tissue forceps and raised from its base, the laser knife "optical fiber tip" was handled perpendicular to the surgical site. The excision procedure started from the base of the mass. The specimens were inserted in $10 \%$ formalin solution as a fixative material and sent for histopathological examination.

Safety glasses were worn by the patient, the surgeon and the assisting staff all through surgery.

The wounds in the oral mucosa were not sutured and healing was facilitated by granulation and secondary epithelialization. After surgery, analgesic medication and antimicrobial mouthwash were prescribed to all patients to shield the wound initially and prevent any secondary infection.

Case 1: A 48 years old healthy man referred to our department with an oval and painless mass in the lower lip vermilion. The lesion was pedunculated and measured about $1 \mathrm{~cm} \times 1 \mathrm{~cm}$ in size (Fig.1). The tumor was excised under local anesthesia and the tip was applied continuously in contact mode to the tissue (Fig.2 and 3). Histopathological examination diagnosed it as pyogenic granuloma. No bleeding, swelling or scar tissue formation was observed (Fig. 4).

Case 2: A male patient aged 57 years presented with a nontender, pedunculated, oval tumor arising above the top of the left mandibular edentulous ridge and measuring about $4 \mathrm{~cm} \times 1 \mathrm{~cm}$ in size. The surface of the lesion was smooth with a normal color, and had a fibrotic consistency (Fig.5). The tumor was excised at the 
base under local anesthesia (Fig.6 and 7). Histopathological examination diagnosed it as peripheral ossifying fibroma (Fig.8).

Case 3: A healthy female patient, aged 48 years old referred to us with a jugal nodule in the right cheek. The nodule was painless, sessile with a firm consistency and measuring $1 \mathrm{~cm} \times 1 \mathrm{~cm}$ (Fig.9). Excisional biopsy was done under local anesthesia and histopathological examination diagnosis was an irritational fibroma (Fig.10, 11 and 12).

Case 4: A 32 years old male patient presented a soft mass in the lateral border of the tongue; the lesion was sessile, painless and the covering mucosa was normal (Fig.13). The tumor was excised under local anesthesia (Fig.14), and the histopathological diagnosis was a peripheral fibroma (Fig. 15 and 16).

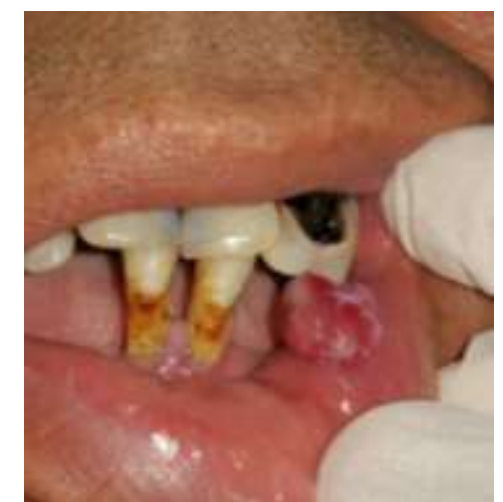

Figure 1: The clinical feature of the labial lesion
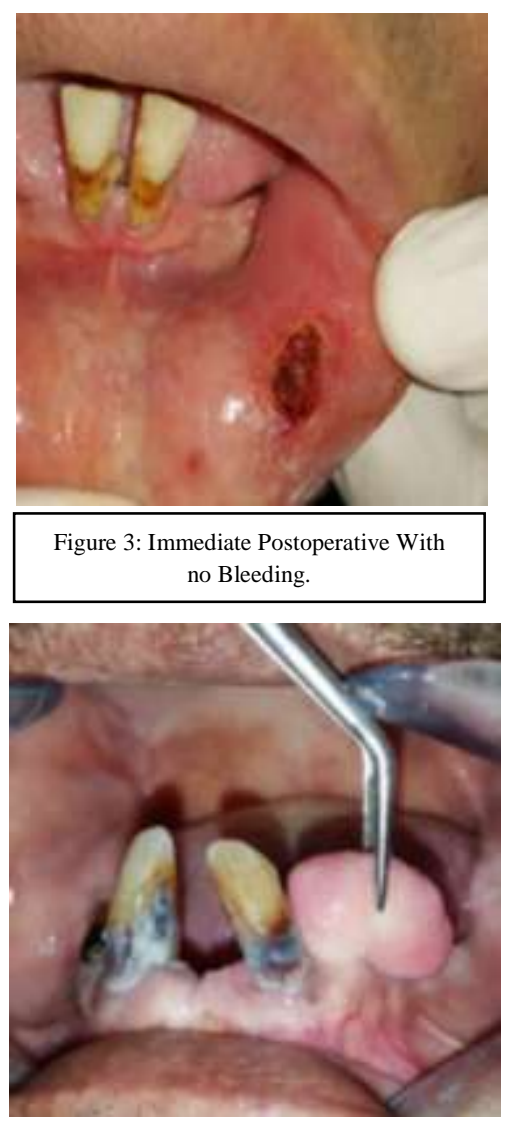

Figure 5: Clinical feature of the tumor

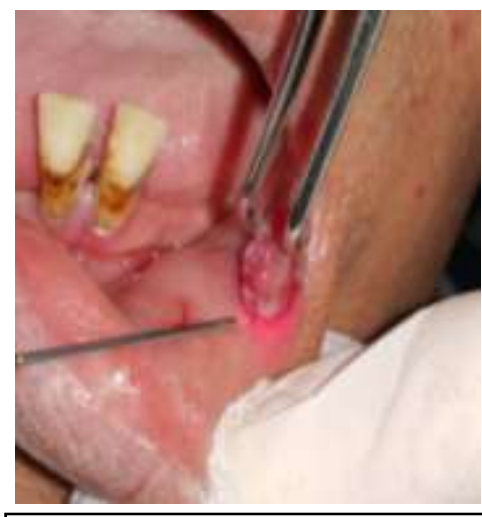
Figure 2: Application of the diode laser and
view of guiding light during operation
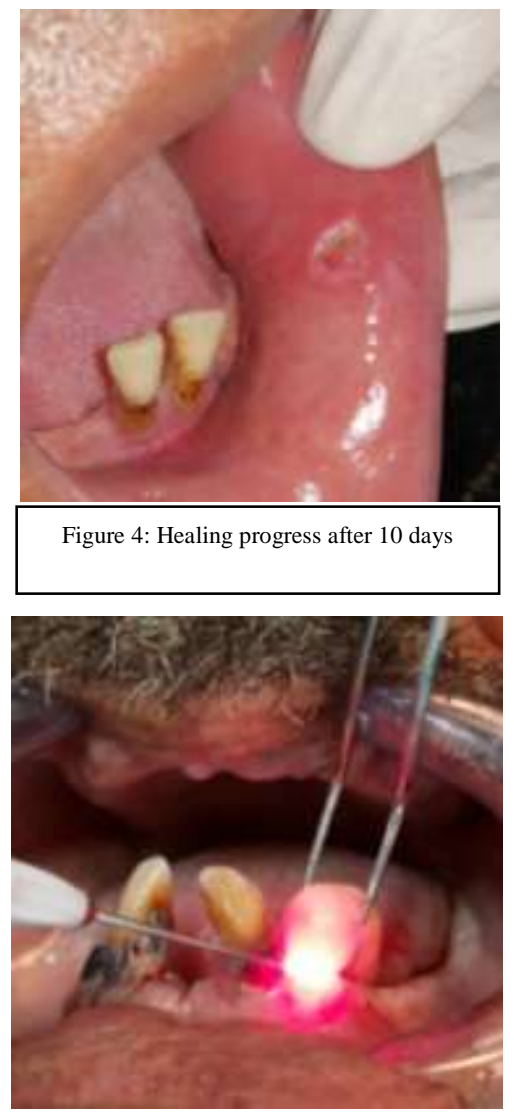

Figure 6: Application of the diode laser and view of guiding light during operation 

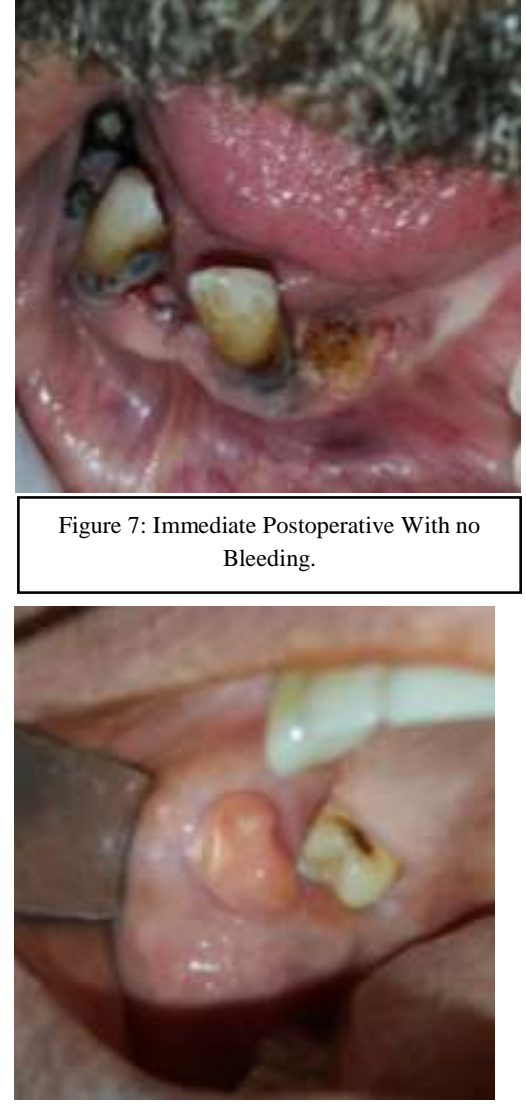

Figure 9: Clinical feature of the jugal nodule

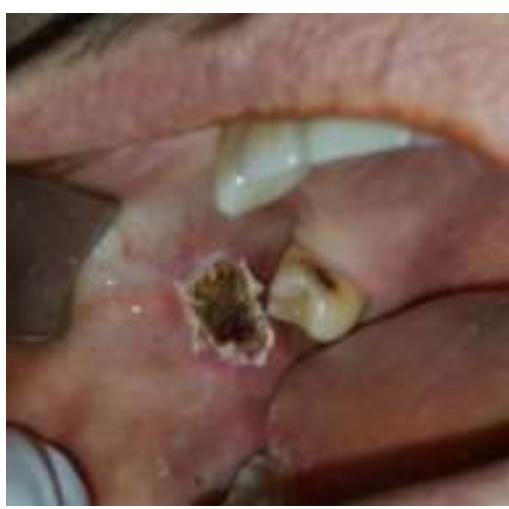

Figure 11: Immediate Postoperative With no Bleeding.

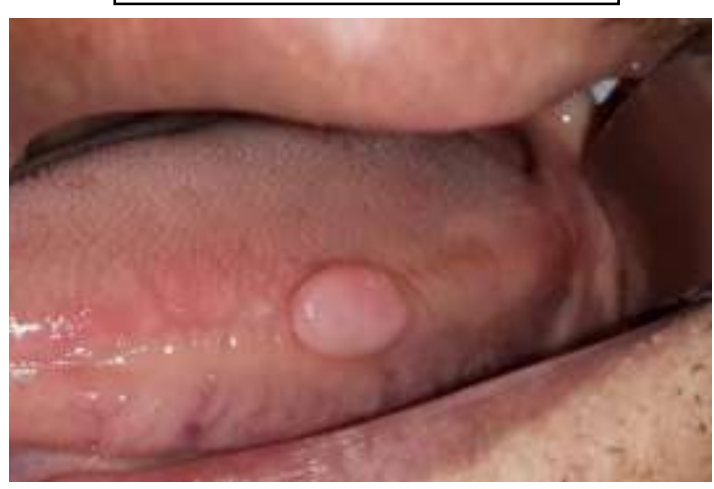

Figure 13: Clinical feature of the tumor

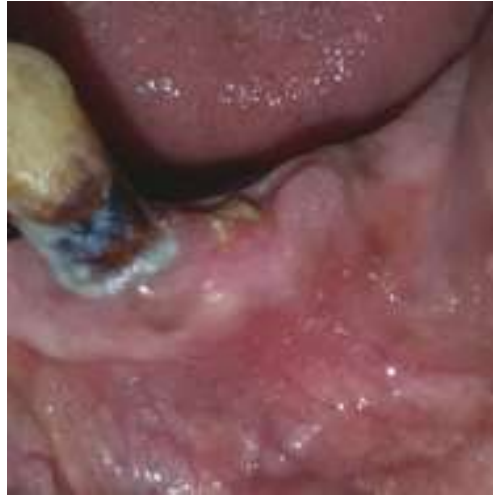

Figure 8: Complete wound healing without scar formation after 2 weeks

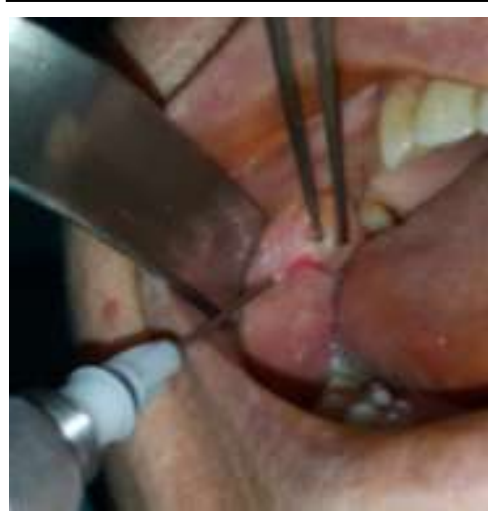

Figure 10: Application of the diode laser an view of guiding light during operation

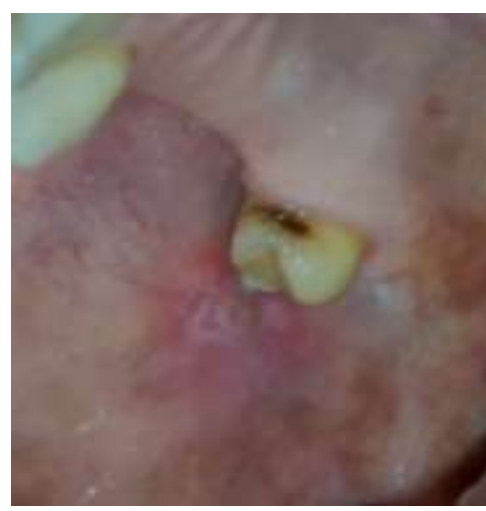

Figure 12: Two Weeks Postoperative Demonstrating Adequate Healing.

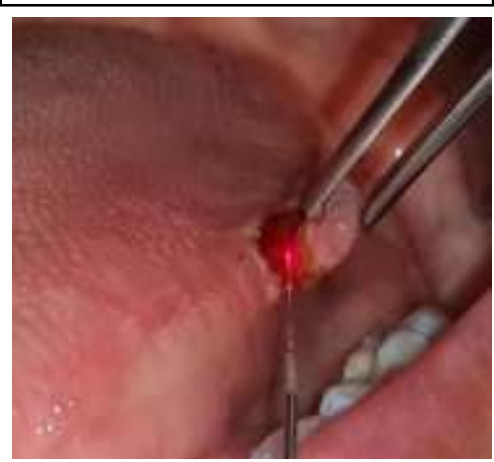

Figure 14: Application of the diode laser and view of guiding light during operation 

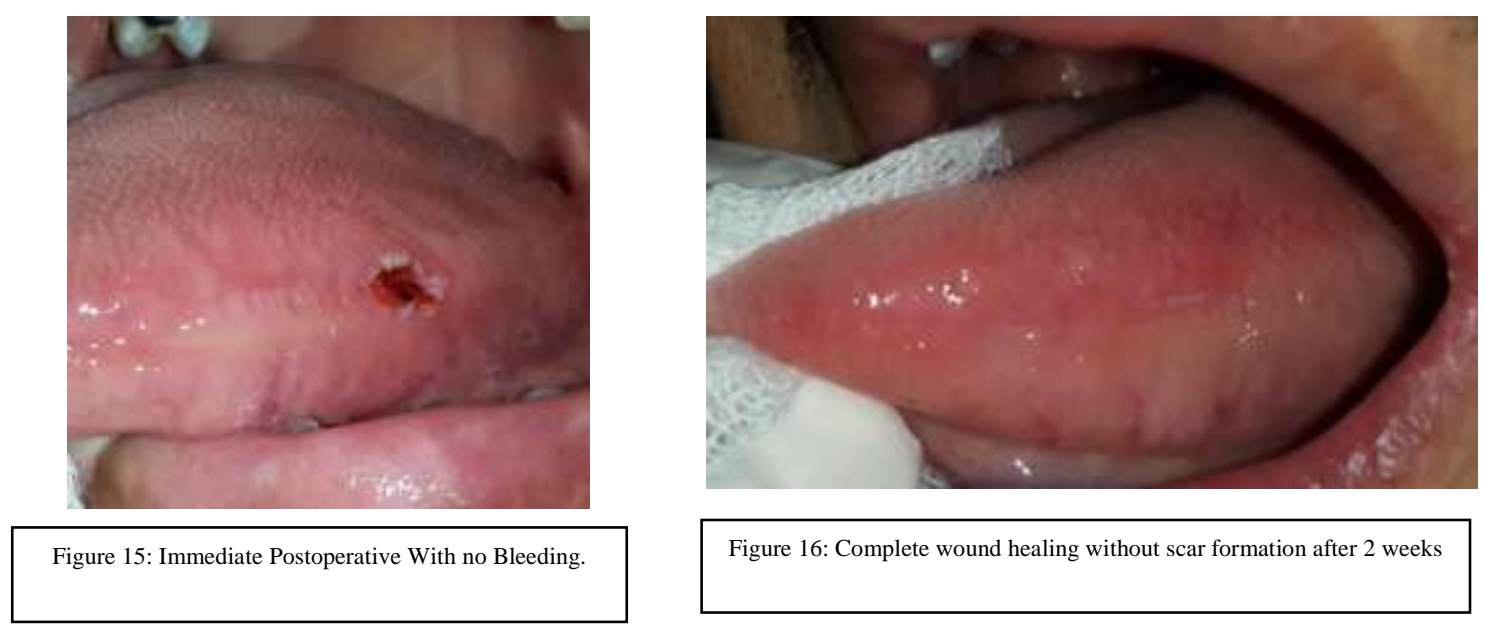

Figure 16: Complete wound healing without scar formation after 2 weeks

\section{Discussion}

Laser systems and their application have made enormous progress in dentistry and especially the field of oral surgery as collateral approach for soft tissue surgery. This rapid progress can be attributed to the fact that lasers allow efficient execution of soft tissue procedures with excellent hemostasis and field visibility. When matched to scalpel, electrocautery or high frequency devices, lasers offer maximum postoperative patient comfort $[9,10]$.

Common lasers used in oral surgeries are $\mathrm{CO}$, Er. family, diode and Nd:YAG. Also low level lasers are used in assisting the procedures of disinfection and healing.

CO2 was the first laser introduced to dental practitioners in the mid-1980s, because of its outstanding cutting abilities and surprisingly [11]. It has a wavelength of $10,600 \mathrm{~nm}$ and is readily absorbed by water. Therefore it does not penetrate too deep into the tissues $(0.1-0.23 \mathrm{~mm})$ without repeated or prolonged use. This is used ideally for superficial lesions, resurfacing of the skin and removal of sialoliths [8].

The Erbium family lasers (Er:YAG 2940nm and Er,Cr:YSGG 2790nm) are the lasers mainly used in dentistry for cavity preparation [11]. Their emission wavelengths are perfectly matched to the absorption maximum of water which is a component of oral tissue [12].

Nd:YAG lasers: This is mostly used in hair removal procedures. In addition, it is also used in removing tattoos and certain pigmented lesions. It has a wavelength of $1064 \mathrm{~nm}$ and is frequently used in cartilage and bone ablation [8]. Its deep extinction length and penetration depth in soft tissue create significant lateral tissue damage, desirable for vascular lesions, but not for other pathology $[1,12]$

Diode laser (810-1064 $\mathrm{nm}$ ) has become very popular in general dentistry because of their small size, low cost, fiber optic delivery and ease of use for minor surgery of oral soft tissue. The use of low level Diode laser for noninvasive athermal laser therapy is popular in European countries [1,12].

The excision of oral mucosa lesions is one of the frequent uses of this tool. The advantages of laser surgery are relatively hemostasis and outstanding field visibility, minimal swelling, scarring and coagulation, no need for suturing, reduction in surgical time and less or no postoperative surgical pain and edema. Also, the laser instantly disinfects the surgical wound as well as allowing a noncontact type of operative procedure and therefore no mechanical trauma to the tissue $[3,9]$.

Patients undergoing diode laser surgery in the oral cavity seem to have less pain because the thermal necrosis created by tissue vaporization cause sealing of the ends of sensory nerves, decrease their ability to transmit stimuli [13,14], also denaturation protein act a decreasing pain [15].In addition, all the immune reaction component present the range of diode laser treatments antigen, antibodies, cytotoxic protein and sub-epithelial lymphocyte are all denaturated due to its deeper penetration. That means destruction of the diseased epithelium with its surface antigen, less risk of edema and the dressing layer of denaturated protein enhance healing with less risk of secondary infection [16].

Koppolu et al on 2014 [17] compared the excision of lesions with diode laser and scalpel and concluded that for intraoral soft tissue surgical techniques, laser is a reasonable alternate to the scalpel. Diminished postoperative swelling and pain is a distinctive quality of lasers and facilitates improved safety when performing surgery within the airway [9].

Studies evaluating the thermal tissue effects of diode lasers are not decisive. In an experimental study of Goharkhay et al on 1999[18], histological analysis to confirm vertical and horizontal tissue damage as well as incision depth and width was performed in the oral mucosa using $810 \mathrm{~nm}$ diode laser, and subsequently the 
authors concluded that the outstanding cutting ability and the tolerable damage zone visibly showed the diode laser to be very efficient and a valuable option in soft tissue surgery in oral cavity.

Diode laser reveals a bactericidal effect and help to reduce inflammation in oral and dental treatment which decreasing the incidences of infection [19]. Our $3^{\text {rd }}$ case was suffering from infection and pain persisted until the end of ten days due to fact that the patient did not follow our instructions. When the laser can be used to vaporize the base of the lesion, destroying cells layer by layer and healing of the underlying minor salivary glands, recurrence is reduced, obviating that need for excision of the minor glands [20].

\section{Conclusion}

We conclude that lasers are rapidly becoming the standard of care in contemporary dental practice and have been shown to be superior to conventional mechanical approaches and to be used as alternative instruments for excisional biopsies of oral soft tissue lesions. Intra- and postoperative complications are rare, with minor bleeding being the only complication observed during surgeries.

Practitioners should be satisfied that novel clinical approaches have a sound scientific basis, and are not adopted solely just on the basis of anecdotal reports or incomplete research.

There are no acknowledgements.

\section{Acknowledgements}

\section{References}

[1]. Asnaashari M, Zadsirjan S. Application of Laser in Oral Surgery. J Lasers Med Sci 2014;5(3):97-107.

[2]. Asnaashari M, Mohebi S, Paymanpour P. Pain reduction using low level laser irradiation in single-visit endodontic treatment. J Lasers Med Sci 2011;2(4):139-43.

[3]. Azma E, Safavi N. Diode Laser Application in Soft Tissue Oral Surgery. J Lasers Med Sci 2013; 4(4):206-11.

[4]. D'Arcangelo C, Di Nardo Di Maio F, Prosperi GD, Conte E, Baldi M, Caputi S. A preliminary study of healing of diode laser versus scalpel incisions in rat oral tissue: a comparison of clinical, histological, and immunohistochemical results. Oral Surg Oral Med Oral Pathol Oral Radiol Endod 2007; 103: 764-73.

[5]. Brandon MS, Strauss RA. Complications of CO2 laser procedures in oral and maxillofacial surgery. Oral Maxillofac Surg Clin North Am 2004;16: 289-99.

[6]. Coluzzi DJ. Lasers in dentistry. Compend Contin Educ Dent 2005;26(6A Suppl):429-35.

[7]. Boj JR, Poirer C, Hernandez M, Espassa E, Espanya A. Review: Laser soft tissue treatments for paediatric dental patients. Eur Arch Paediatr Dent 2011;12(2):100-5.

[8]. Abraham RJ, Lankupalli AS. Laser Management Of Introral Soft Tissue Lesions - A Review Of Literature. IOSR-JDMS, Volume 2014; 13(1): 59-64.

[9]. Mathur E, Sareen M, Dhaka P, Baghla P. Diode laser excision of oral benign lesions. J Lasers Med Sci. 2015;6(3):129-132.

[10]. Coluzzi DJ. Fundamentals of dental lasers: Science and Instruments. Dent Clin Am. 2004;48:751-770.

[11]. Paghdiwala AF. Application of the erbium: YAG laser on hard dental tissues; Measurement of the temperature changes and depth of cut. Lasers Med Surg Dent 1988;64:192-201.

[12]. Convissar RA. Principles and practice of laser dentistry: Mosby; 2010.

[13]. White JM, Goodis HE, Rose CL. Use of pulsed Nd:YAG laser for intra-oral soft tissue surgery. Lasers Surg Med 1991; 11: $455-561$.

[14]. Gold SI, Vilardi MA. Pulsed laser beam effects on gingival, J Clin Periodontal 1994; 21: 391-396.

[15]. Mona S, Ahmed ELK, Ali S. Management of oral lichen planus using diode laser (980nm): A clinical study. Egyptian Dermatology online J. June 2005; 1(1): 3.

[16]. Mortiz A, Schoop U, Gharkhay K, Wermisch J, Sperr W. treatment of periodontal pockets with a diode lasers. Laser Surg Med 1998; 22: 302-311.

[17]. Koppolu P, Mishra A, Kalakonda B, Swapna LM, Bagalkoikar A, Macha D. Fibroepithelial polyp excision with laser and scalpel: A comparative evaluation. Int J Curr Microbiol App Sci. 2014;3(8):1057-1062.

[18]. Goharkhay K, Moritz A, Wilder-Smith P, et al. Effects on oral soft tissue produced by a diode laser in vitro. Lasers Surg Med. 1999;25(5):401-406.

[19]. Wilder-Smith P, Pang J, Kuroseki T Investigating the range of surgical effects on soft tissue produced by carbon dioxide laser. JAM Dent Associ. 1997; 128: 583-588.

[20]. Yueb H, Chum C, Hu-Hsun K, Philip W. Treatment of mucocele of the lower lip with carbon dioxide laser. J Oral MaxillofacSurg, 2007; 65: 855-858. 